\title{
Recommended housing conditions and test procedures can interact to obscure a significant experimental effect
}

\author{
MARK LYTE and NOEL OPITZ \\ Minneapolis Medical Research Foundation, Minneapolis, Minnesota \\ LISA E. GOEHLER and RONALD P. GAYKEMA \\ University of Virginia, Charlottesville, Virginia \\ and \\ J. BRUCE OVERMIER \\ University of Minnesota, Minneapolis, Minnesota
}

\begin{abstract}
Routine animal husbandry variables, such as group housing of mice and the order of testing of cagemates, are currently viewed to be essentially neutral with respect to the outcome of most, if not all, animal-based experiments, including those that utilize behavioral measurements. During the course of experiments that have utilized the elevated plus-maze to examine the ability of a bacterial challenge of mice to induce anxiety-like behavior, due to the activation of various cytokine pathways, we followed the recommendation of laboratory animal care staff to house the mice in pairs. When we tested the members of the pairs successively, it was found, for the first experimental set, that the behavior that reflects anxiety (time in closed arms) of the first-tested animal differed from that of the second-tested animal for both the experimental and the control animals and, critically, that these changes were in the opposite directions for the controls and the experimental animals, thus obscuring the effect of the experimental manipulation. A second, independent experimental set also obtained a significant effect for the order of testing effect in the bacterial-challenged group, but not in the saline control group, although a similar trend was evident in this group as well. These results indicate that special care should to be taken in implementing housing recommendations and that preliminary tests may be necessary to ensure that housing conditions do not interact with tests of the phenomenon under experimental investigation.
\end{abstract}

Factors outside of any specific experimental manipulation-specifically, housing and order of testing of subjects within a specific cage - are believed to be neutral with respect to behavioral measure outcome. During the course of experiments in our laboratory in which we have sought to examine the ability of subclinical gastrointestinal bacterial infection to influence behavior (Gaykema, Goehler, \& Lyte, 2004; Lyte, Varcoe, \& Bailey, 1998), we have observed that such neutral factors may, in fact, not be neutral at all but, instead, may significantly impact experimental outcome. The cumulative effect of not recognizing the possible contribution of such factors as housing and order of testing may be that the behavioral effects of the specific experimental manipulation under study, which in our case was subclinical challenge of animals with a gastrointestinal food-borne pathogen, are obscured.

This investigation was supported by National Institutes of Health Grant MH50431 to M.L. Correspondence concerning this article should be addressed to M. Lyte, Department of Pharmacy Practice, School of Pharmacy, Texas Tech University Health Sciences Center, 3601 4th Street, STOP 8162, Lubbock, TX 79430-8162 (e-mail: mark.lyte@) ttuhsc.edu).
It is well established that infections may result in an alteration of behavior, presumably due to the induction of immune-related products, such as the proinflammatory cytokines, which can interact with various neuronal processes, leading to behavioral alterations known collectively as sickness behavior (Kent, Bluthe, Kelley, \& Dantzer, 1992; Larson, 2002). Using an ecologically relevant model of gut infection by a common food-borne bacterial pathogen, Campylobacter jejuni, we have been testing the hypothesis that certain behaviors may be modulated, in part, by infectious microorganisms within the gut through mechanisms involving visceral sensory signaling from the gut to the brain, often referred to as the gut-to-brain axis. Prior work has shown that a subclinical infection in mice with $C$. jejuni results in the production of anxiety-like behavior, as measured on the elevated plus-maze (EPM), without detectable systemic immune activation that otherwise could account for this behavioral change (Lyte et al., 1998). We have further shown that in mice, infection with C. jejuni leads to neuronal activation within specific brain regions that may shed light on the neurocircuitry implicated in anxiety-like behavior (Gaykema et al., 2004). As such, the present naturalistic model of bacterial infection points to mechanisms underlying behavioral change that 
differ from those responsible for sickness behaviors occurring in parallel with systemic inflammation (Dantzer, 2001; Kent et al., 1992). A link between gastrointestinal infections and more subtle anxiety-like effects, rather than full-blown sickness, may therefore represent a novel and, as yet, unidentified mechanism involving a gut-to-brain pathway that plays a significant role in regulating behavior, likely via changes in affect and/or motivational states. The urgency to understand this infection-behavior link is great, since such gastrointestinal infections are quite common in the human population (Lacey, 1993; Roberts, 1990).

In seeking to elucidate the mechanisms by which a gut-to-brain axis may mediate the ability of per orally acquired infections to influence behavior, we have employed a number of behavioral measures; principal among these has been the EPM, wherein entries into closed arm areas and the time spent there are taken as indices of anxietylike behaviors. This report concerns itself with our experiences in this research that can aid other investigators in avoiding experimental noise (e.g., Enserink, 1999; Wahlsten et al., 2003).

Today, laboratory animal welfare is of special concern because we have learned that healthy animals provide the best experimental models for testing hypotheses that involve subtle behavioral changes that are sensitive to both external (environmental) and internal (immune and hormone) status. Toward this end, laboratory animal veterinarians and care providers properly make many suggestions that may improve the health status of the animals (see, e.g., Olsson \& Dahlborn, 2002; Porter, Scott, \& Walker, 1970). Among the recommendations commonly made are for group - or at least, pair-housing (e.g., Love, 1994; Simmel \& Walker, 1972), with the assumption that this yields a healthier animal, although the data are in fact mixed (e.g., Rao \& Lindsey, 1988; Weinreich, Hoebe-Hewryk, Boog, \& Ivanyi, 1996). We received just such guidance to house our animals in pairs, guidance which we heeded. As we will reveal below, the effect was to impair experimental efficiency to the point of obscuring the experimental phenomenon under study.

\section{METHOD}

\section{Subjects}

Thirty-six 5-week-old CF-1 male mice were purchased from Charles River Laboratories (Wilmington, DE). Upon receipt, the animals were housed at a density of 2 animals per cage (width, $16 \mathrm{~cm}$; length, $22 \mathrm{~cm}$; height, $13 \mathrm{~cm}$ ) and were placed under a reversed day:night light cycle (dark, $0400-1600$ h; light, $1600-0400$ h). All the animals were maintained in the animal facility for 5 weeks to allow for acclimatization before use in experimental protocols. The animals were fed ad lib and had free access to water. All treatment procedures were approved by the Minneapolis Medical Research Foundation Institutional Animal Care and Use Committee.

\footnotetext{
Apparatus and Procedure

Bacterial challenge. Per oral challenge of animals with C. jejuni was conducted essentially as has previously been described (Lyte et al., 1998). Two days before per oral challenge, a stock culture of C. jejuni (No. 29428, American Type Culture Collection, Bethesda, $\mathrm{MD})$ was plated onto BBL Campylobacter agar plates containing
}

10\% sheep blood plus 5 antimicrobials (BBL 21727, Becton Dickinson, Cockeysville, MD) and was incubated under microaerophilic conditions (BBL Campy Pak Plus 72045 ) at $37^{\circ} \mathrm{C}$. On the day of challenge, individual colonies were removed from the plate surface and were resuspended in sterile saline, followed by a $2 \times$ wash. The optical density of the bacterial solution was measured (at A550), plotted against a known standard curve to yield colony-forming units, and adjusted to yield approximately $1 \times 10^{9}$ bacteria per milliliter. Using a ball-tipped, stainless steel feeding needle, the mice were per orally inoculated with $0.2 \mathrm{ml}$ of $C$. jejuni suspended in saline (approximately $1-2 \times 10^{8}$ bacteria/mouse) or saline alone at $1000 \mathrm{~h}$. The animals were monitored daily for changes in food consumption and water intake, as well as for alterations in overt behavior, such as grooming. No alterations in the preceding parameters were noted, nor was any evidence of diarrhea observed. Previous work has demonstrated that animals inoculated with this amount of C. jejuni do not evidence any signs of sickness behavior or, upon post mortem, show any evidence of enlarged Peyer's patches, lymph nodes, or other signs of inflammation (Gaykema et al., 2004; Lyte et al., 1998).

Elevated plus-maze. The basic construction of the EPM has been described in detail elsewhere (Lister, 1987; Schrott \& Crnic, 1996). In brief, the maze was constructed of two open arms (35-cm length $\times 5$-cm width) and two closed arms of the same dimensions. The EPM apparatus employed in our study was a commercially available system from Hamilton-Kinder (Poway, CA) that employed pairs of photocells distributed along both open and closed arms at approximately $5-\mathrm{cm}$ intervals (Figure 1A). The open and closed arms were oriented at right angles to each other and extended from a central platform of $5 \times 5 \mathrm{~cm}$, which gives the device the overall shape of a large plus sign (Figure 1A). The open arms, center platform, and floor were constructed of black Plexiglas, and the walls of the closed arms were made of clear Plexiglas $15 \mathrm{~cm}$ in height. To the open arms, a raised edge of 0.5 - $\mathrm{cm}$ height (Rodgers \& Cole, 1993 ) and slightly textured rubber flooring (3M tread cover, St. Paul, MN) were added to facilitate gripping. In addition, the EPM was elevated $62 \mathrm{~cm}$ above the floor. When placed onto the center platform of the EPM at the start of the behavioral measurement, the mouse may choose to enter any one of the four arms. All the equipment around the maze was covered with white fabric to match the uniform whiteness of the walls and floor. Illumination of the maze was obtained from two oppositely placed, 25-W photographic red bulbs approximately $1.5 \mathrm{~m}$ from the testing enclosure and approximately $45^{\circ}$ above the apparatus. Movement on the maze was detected by interruption of infrared photobeams between pairs of photocells (Figure 1B) and was recorded by a dedicated data acquisition program (Motor Monitor, Hamilton-Kinder), which then allowed for various behaviors (entry into arms, time spent in specific areas) to be analyzed.

Behavioral testing. All the mice were tested between 9:00 a.m. and 12:00 p.m., the approximate midpoint of their dark cycle and 24-48 h postchallenge with $C$. jejuni or saline vehicle. All the equipment was turned on and prepared prior to introducing any animals to the testing room. The EPM was cleaned with $20 \%$ isopropyl alcohol solution prior to and between test runs. The home cage of a pair of animals was then brought into the testing room, one cage (containing one pair) at a time. The first mouse was tested on the maze immediately, while the second mouse remained in the homecage on a nearby table. The individual conducting the testing was concealed behind a barrier and was not seen by either animal; nor could the animals see one another. Following the 5-min testing period of the first cagemate, the first mouse was returned to the homecage while the maze was cleaned with a $20 \%$ isopropyl alcohol solution and thoroughly wiped dry (approximately $3 \mathrm{~min}$ ). Since the same cleaning solution was used during the course of the entire experiment - including prior to placing the first animal on the EPM, immediately following its removal, and prior to the second mouse's being tested - any residual alcohol odor would have been equivalent 
A

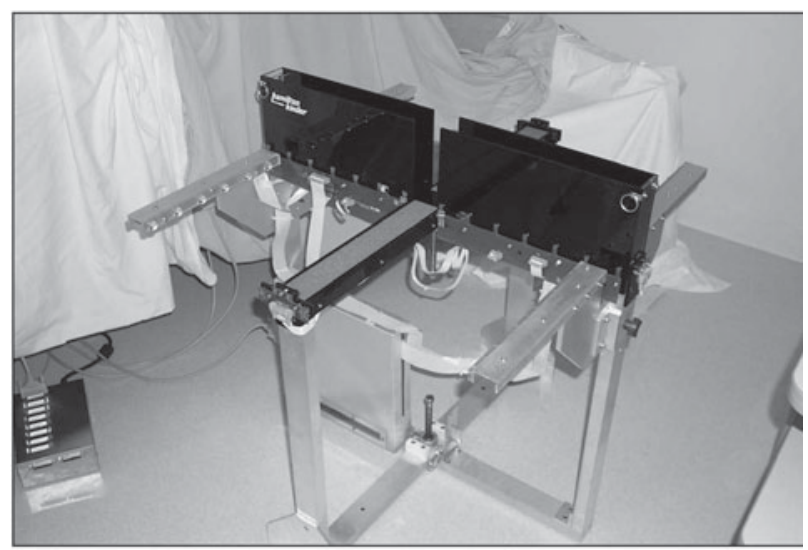

B

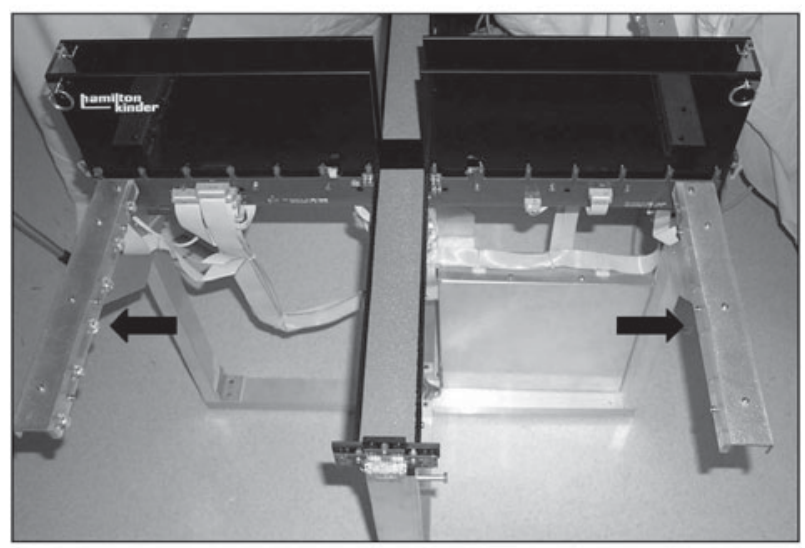

Figure 1. (A) The automated elevated plus-maze (EPM; Hamilton-Kinder, Poway, $\mathrm{CA}$ ) consists of two open and two closed arms, each with 16 electronic beams placed along the length of each arm, which are used to record the animal's movements in the maze ( 2 additional beams capture the subject in the intersection of the two arms). As is described in the Method section, the test animal is placed in the center intersection and remains on the EPM for a total of $5 \mathrm{~min}$, with movement data (beam breaks) along the arms directly downloaded into a computer and analyzed by a dedicated software package (Motor Monitor, Hamilton-Kinder, Poway, CA). At the bottom left is the data transfer unit that collects data from the EPM and downloads directly to a computer (not pictured) in real time. (B) Close-up view of the photocell arrangement on one of the open EPM arms, which records movement upon breakage of the infrared beam by the animal. The open arm (with raised ridges) is located between the two photocell-containing arms. A photocell pair is indicated on the left and right stainless steel arms by arrows in the figure.

throughout, thereby making it unlikely that alcohol-related odor significantly affected results relating to the order of testing. The second mouse was then removed from the homecage and immediately tested in the same manner and for the same 5-min duration as the previous mouse. After testing, this second animal was returned to its cage, and the apparatus was again cleaned as described above. The cage was then returned to the adjacent animal holding room, and the next homecage and its pair were brought into the testing room. This procedure was repeated for all the remaining animals.
In order to obviate possible confounding factors, such as counterbalancing of the test schedule between groups, testing was alternated between pairs of $C$. jejuni-challenged animals and pairs of saline controls. For example, one cage ( 2 animals) of infected animals was tested, followed by one cage ( 2 animals) of control animals. The apparatus was cleaned just prior to the testing of each individual animal to avoid any wash-time-to-run-time delay. The animals were brought one cage at a time from the holding room to an adjacent testing room, which housed the EPM. Once the animals were in the room, the apparatus was cleaned, and the first animal was tested immediately following the cleaning. After testing, the animal was returned to the cage, and the apparatus was cleaned again in an identical manner. The second animal (cagemate) was then immediately tested. The reason for this procedure was to avoid any wash-time-torun-time differences for first and second animals. When the second animal finished testing, it was returned to the cage, and the cage was returned to the holding room. The next cage (pair of animals) was then brought into the testing room, and the animals were tested following the same procedure. The exact schedule, which was maintained for all the animals, ensured that there were no differences in testing methodology between groups. We therefore believe that no additional confound was present, including that of counterbalancing, that may have differentially affected one group over the other.

\section{RESULTS}

The primary behavioral data of this experimental research were each mouse's distribution of test time spent in the open and closed areas of the EPM. The number of entries into and the amount of time spent in the closed arms were our focus, because the more time spent in the closed arm (and inversely, the less in the open arms), the greater the inferred anxiety-like status of the mouse (see Bhattacharya, Mohan Rao, \& Sen, 1995; Privette \& Terrian, 1995; Zhukov \& Vinogradova, 1995). The data for these two measures are shown in Figures 2 and 3, which report observations from two independent experiments, each containing a saline control and a $C$.jejuni-challenged arm. Data were submitted to a two-way ANOVA, using the Prism 4 statistical program (GraphPad Software, San Diego). In all tests, a level of $p<.05$ or better was interpreted as an indication of significance.

As Figure 2A shows, the first- and second-tested cagemates of both the saline-treated control and the $C$. jejuni treated experimental groups responded differently in terms of the number of closed arm entries. The first-tested control mice made fewer entries than did the second, whereas in contrast, the first-tested experimental infected mice made more entries than did the second-tested; indeed, there was a clear crossover effect. A $2 \times 2$ ANOVA yielded a significant interaction between order of testing and treatment group $[F(1,12)=13.76, p=.003]$, resulting in no overall effect of experimental treatment. Indeed, the interaction and order of testing together accounted for more than $45 \%$ of the total variance. There was not a significant main effect of the experimental treatment (prior challenge with $C$. jejuni). The experiment was repeated, and the results are shown in Figure 2B. Whereas a significant order of testing effect was observed in the $C$. jejuni-challenged animals, thereby replicating the results 


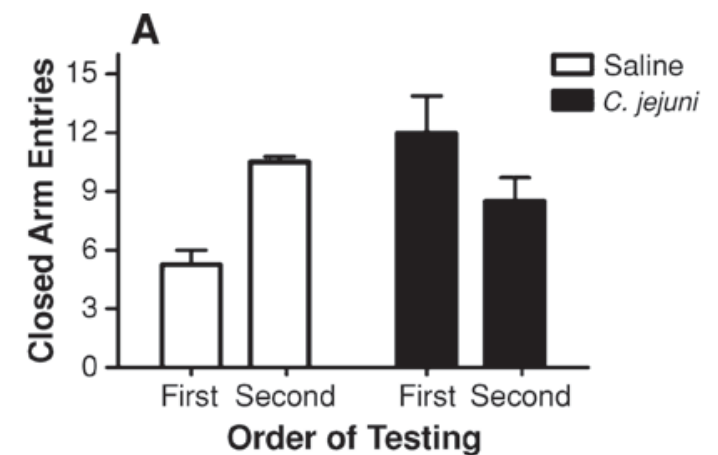

spent more time in the closed arms than did the secondtested (Figure 3B). However, it was not observed that the first-tested control (saline-challenged; Figure 3B) mice spent less time in the closed arms than did the second, due to the 2 saline outlier mice, as was described previously. This resulted in a $2 \times 2$ ANOVA that did not yield a significant interaction between order of testing and treatment group $[F(1,16)=3.53, p=.0787]$ but did result in a significant order effect $[F(1,16)=4.59, p=.0480]$. For Figure $3 \mathrm{~B}$, the interaction and order of testing accounted for more than $50 \%$ of the variance. Due to the variability in the saline group, a significant effect of the experimental treatment with $C$. jejuni overall was obtained $[F(1,16)=$ 7.47, $p=.0147]$.

For the results shown in Figures $2 \mathrm{~A}$ and $3 \mathrm{~A}$, but not Figures $2 \mathrm{~B}$ and $3 \mathrm{~B}$, both measures, the tests of the second cagemates from the control and experimental animals completely obliterated a significant effect of saline versus C. jejuni treatment detectable in the tests of the first cagemates $[t(12)=4.04$ and 3.53, respectively; both $p$ s $<$ $.01]$. Had order of testing not been entered as a factor, as might be done if one thought it not relevant, we would have missed the important experimental effect. For all the groups shown, including the saline group in Figures 2B and $3 \mathrm{~B}$, differences in order of testing between first and second animals were consistently obtained for all cagemates.

In addition, we wish to note that although it is permissible, and frequently desirable, to combine the data from identical experiments, we have opted not to do so in this case. Combining the data from the two experiments would neutralize the order effect in the saline-treated group. This would occur because of the results obtained from just 2 of the control animals, described above for Figure 3, which there is substantial reason to believe are outliers. When viewed separately, one can easily ascertain how closely the two experiments mirror one another, whereas combining the results would allow the anomalous test results of only 2 animals to obscure and negate a potentially significant finding.

Furthermore, an additional experiment was performed utilizing another gram-negative intestinal pathogen, Citrobacter rodentium, in a manner identical to that described for $C$. jejuni. Preliminary data obtained with $C$. rodentium have demonstrated a pattern similar to that seen with $C$. jejuni, thus suggesting that these results may be generalized to other pathogens. Although there were no outliers in the $C$. rodentium trial, the values obtained via a $2 \times 2$ ANOVA were just shy of statistical significance $[F(1,12)=4.590, p=.0534]$ for both order and testing. Although not statistically significant, we mention these results here simply to demonstrate that the pattern is consistent, even when an alternate pathogen is employed.

\section{DISCUSSION} $.72, p=.0167]$. Again, the interaction and order of testing accounted for more than $50 \%$ of the variance. The effect of the experimental treatment overall was not significant. Similar to the results shown in Figure 3A, the first-tested experimental (C. jejuni-challenged) mice

The present results reveal how sensitive tests of anxietylike behaviors are to the interaction of pair-housing con- 

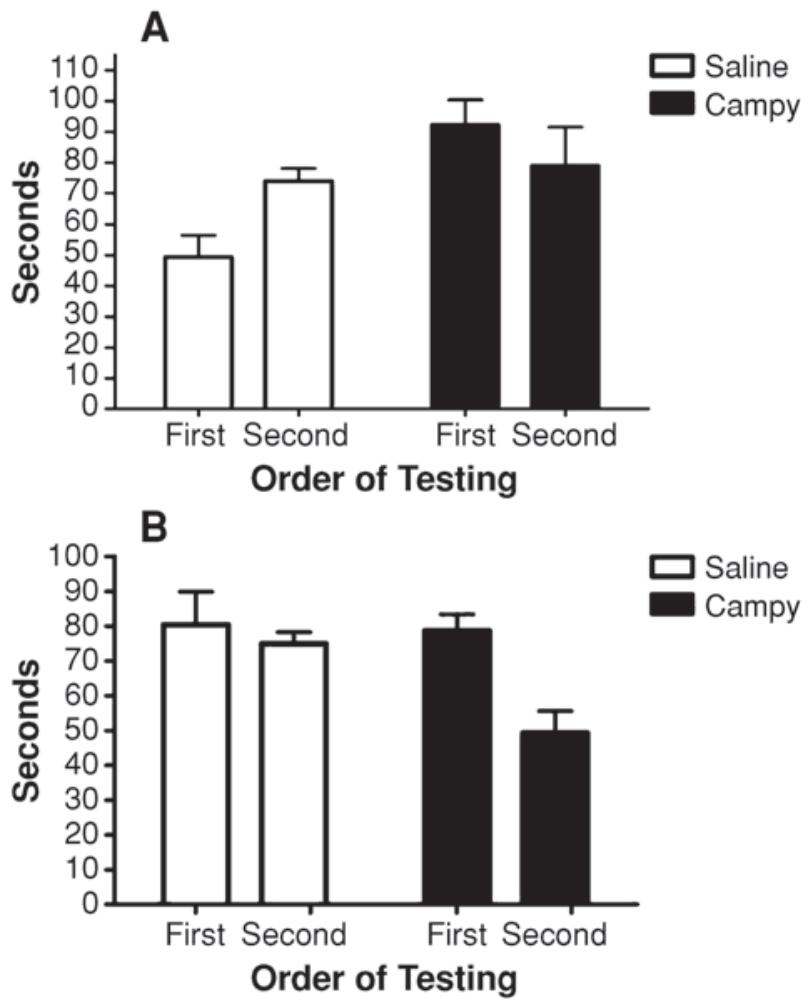

Figure 3. Time spent on the closed arms of the elevated plusmaze by animals per orally challenged $24 \mathrm{~h}$ prior to testing. Results shown are for two independent experiments: (A) saline, $n=$ 8 , and $C$. jejuni, $n=8$; (B) saline, $n=10$, and $C$. jejuni, $n=10$. Paired bars indicate experimental variation observed between animals during testing. The time between the testing of the first animal from the pair housed in a cage and the placing of the second animal on the elevated plus-maze was approximately $8 \mathrm{~min}$ (5 min for testing on the maze and $3 \mathrm{~min}$ for cleaning and instrument preparation for the second animal). Results represent the mean $\pm S E M$.

ditions and the consequent experimental procedures for testing the pair. It is important to note that these observations did not result from experiments that were intentionally designed to evaluate housing conditions and order of testing on specific behavioral measures on testing devices such as the EPM. Instead, these experimental findings were, in fact, observed unintentionally and subsequently evaluated for significance in the context of the overall experimental intent to examine the ability of a subclinical bacterial infection to influence behavior.

Clearly, special efforts are required to overcome these intervening factors that obscure the possible effects of controllable variables under study. The EPM is a widely used test of anxiety-like behavior in rodents that has been employed in recent years to assess the effects of treatments that might induce or ameliorate anxiety. The results are not always consistent from laboratory to laboratory, and part of this inconsistency may be attributable to variations in variables judged not important, such as housing, handling, and/or sequence of testing (e.g., Bohannon, 2002). Such judgments are questionable, as the present data illustrate. Indeed, as was mentioned previously, our most recent experiment serves to underscore the potential significance of these findings, in that the pattern was repeatable under identical housing and experimental conditions despite the use of a completely different pathogen. We hope our experiences will serve to alert researchers to the importance of such factors and will help them to have open dialogues with their laboratory animal care staff to ensure the balance between animal welfare considerations and the needs of their science.

The present research adds to the growing body of literature on the role of housing as it contributes directly or indirectly to variation in experimental results - with regard to which housing enrichment has been studied the most to date (e.g., Van de Weerd et al., 2002; Wurbel, 2001) —although it is recognized that many other factors are likely to be critical (e.g., Chesler, Wilson, Lariviere, RodriguezZas, \& Mogil, 2002). We appreciate that the dissection of the relevant mechanisms would contribute significantly to the understanding of the observed phenomena and the manner in which treatment manipulations otherwise deemed as neutral can, in fact, affect the outcome of a particular experiment. We hope that this report does stimulate the behavioral community to examine the underlying mechanisms. It is recognized that a certain amount of experimental noise (variations in results due to social deprivation, pheromones, individual differences, etc.) is common and to be expected from nearly any experimental paradigm. However, we have conducted extensive behavioral testing in the past under a variety of environmental conditions. Never before have we encountered the striking patterns observed in these pair-housed animals. Although we cannot identify the source responsible for the inversion of effects described in this article, it clearly does arise from a standard experimental design and widely recommended housing conditions (pairs). The causes remain unknown, but the consequences are clear in that they pose a serious problem for researchers by potentially blocking the detection of significant phenomena. Prior knowledge of the effects of pair housing on experimental outcomes would have enabled us, as well as others who utilize behavior as a tool to assess nontraditional experimental manipulations (such as subclinical bacterial infection), not only to better inform experimental design, but also to realize that the supposed "failure" of any particular manipulation may be due to factors that could mistakenly be considered neutral. Crabbe and Wahlsten (2003) indicated that the degree to which housing conditions, husbandry, and subtle experimental variations cause differences in experimental outcomes is uncertain and that more research is needed to identify relevant factors. The present research contributes to the filling of these specific lacunae.

\section{REFERENCES}

Bhattacharya, S. K., Mohan Rao, P. J. R., \& Sen, A. P. (1995). Anxiogenic activity of intraventricularly administered bradykinin in rats. Journal of Psychopharmacology, 9, 348-354.

Bohannon, J. (2002). Can a mouse be standardized? Science, 298, $2320-2321$ 
Chesler, E. J., Wilson, S. G., Lariviere, W. R., Rodriguez-Zas, S. L., \& MogiL, J. S. (2002). Influences of laboratory environment on behavior. Nature Neuroscience, 5, 1101-1102.

Crabbe, J. C., \& Wahlsten, D. (2003). Of mice and their environments. Science, 299, 1313-1314.

DANTZER, R. (2001). Cytokine-induced sickness behavior: Where do we stand? Brain, Behavior, \& Immunity, 15, 7-24.

EnSERINK, M. (1999). Behavioral genetics: Fickle mice highlight test problems. Science, 284, 1599-1600.

Gaykema, R. P. A., Goehler, L. E., \& Lyte, M. (2004). Brain response to cecal infection with Campylobacter jejuni: Analysis with Fos immunohistochemistry. Brain, Behavior, \& Immunity, 18, 238-245.

Kent, S., Bluthe, R. M., Kelley, K., \& Dantzer, R. (1992). Sickness behavior as a new target for drug development. Trends in Pharmacological Sciences, 13, 24-28.

LACY, R. W. (1993). Food-borne bacterial infections. Parasitology, 107, S75-S93.

LARSON, S. J. (2002). Behavioral and motivational effects of immunesystem activation. Journal of General Psychology, 129, 401-414.

Lister, R. G. (1987). The use of a plus-maze to measure anxiety in the mouse. Psychopharmacology, 92, 180-185.

Love, J. A. (1994). Group housing: Meeting the physical and social needs of the laboratory rabbit. Laboratory Animal Science, 44, 5-11.

Lyte, M., Varcoe, J. J., \& Bailey, M. T. (1998). Anxiogenic effect of subclinical bacterial infection in mice in the absence of overt immune activation. Physiology \& Behavior, 65, 63-68.

Olsson, I. A., \& DAHLBORn, K. (2002). Improving conditions for laboratory mice: A review of "environmental enrichment." Laboratory Animals, 36, 243-270.

Porter, G., Scott, P. P., \& Walker, A. I. (1970). Caging standards for rats and mice: Recommendations by the Laboratory Animal Science Association Working Party of Caging and Penning. Laboratory Animals, 4, 61-66.
Privette, T. H., \& Terrian, D. M. (1995). Kappa opioid agonists produce anxiolytic-like behavior on the elevated plus-maze. Psychopharmacology, 118, 444-450.

RAO, G. N., \& LiNDSEY, J. R. (1988). Ankylosis of hock joints in group caged male B6C3F1 mice. Laboratory Animal Science, 38, 417-421.

Roberts, D. (1990). Sources of infection: Food. Lancet, 336, 859-861.

Rodgers, R. J., \& Cole, J. C. (1993). Anxiety enhancement in the murine elevated plus maze by immediate prior exposure to social stressors. Physiology \& Behavior, 53, 383-388.

SchrotT, L. M., \& CRNIC, L. S. (1996). Increased anxiety behaviors in autoimmune mice. Behavioral Neuroscience, 110, 492-502.

Simmel, E. C., \& Walker, D. A. (1972). The effects of a companion on exploratory behavior in two strains of laboratory mice. Behavior Genetics, 2, 249-254.

Van de Weerd, H. A., Aarsen, E. L., Mulder, A., Kruitwagen, C. L., HENDRICKSEN, C. F., \& BAumans, V. (2002). Effects of environmental enrichment for mice: Variation in experimental results. Journal of Applied Animal Welfare Science, 5, 87-109.

Wahlsten, D., Metten, P., Phillips, T. J., Boehm, S. L., II, BurkhartKasch, S., Dorow, J., ET AL. (2003). Different data from different labs: Lessons from studies of gene-environment interaction. Journal of Neurobiology, 54, 283-311.

Weinreich, S., Hoebe-Hewryk, B., Boog, C., \& Ivanyi, P. (1996). Solitary caging protects mice from ankylosing enthesopathy. Clinical Rheumatology, 15 (Suppl. 1), 32-33.

Wurbel, H. (2001). Ideal homes? Housing effects on rodent brain and behaviour. Trends in Neurosciences, 24, 207-211.

Zhukov, D. A., \& Vinogradova, E. P. (1995). Effects of handling on plasma corticosterone and anxiety depend on coping style of the rat. Fiziologicheskii Zhurnal SSSR im I.M. Sechenova, 81, 93-97.

(Manuscript received May 31, 2004;

revision accepted for publication December 2, 2004.) 